\title{
A Generative Framework to Query Recommendation and Evaluation
}

\author{
Matthew Mitsui \\ 6th Year PhD Candidate \\ Supervisor: Chirag Shah \\ Department of Computer Science \\ Rutgers University \\ New Brunswick, NJ, USA, 08901 \\ mmitsui@cs.rutgers.edu
}

\begin{abstract}
In practice, query recommenders in Web search typically recommend queries directly from a query log or iteratively refine a user's current context to make recommendations. These approaches either limit themselves to queries in the log or do not take necessary exploratory leaps in their recommendations. Moreover, they do not directly incorporate the encompassing, driving information needs and tasks. The author first shows that user queries may not necessarily be the best to use for recommendations and moreover proposes a framework for generating novel queries for query recommendation, using an approximation of need.
\end{abstract}

\section{CCS Concepts}

-Information systems $\rightarrow$ Query suggestion; Personalization; Document topic models; Retrieval effectiveness;

\section{Keywords}

Search session analysis; Diversity; User simulations; Query recommendation

\section{MOTIVATION AND RESEARCH QUES- TIONS}

In the classic machine learning problem of classification, algorithms can be divided into two categories: 1) discriminative methods and 2) generative methods [10]. In the former, a classification algorithm directly finds the best classification label $y$ for a data point $x$ by directly computing $p(y \mid x)$. Generative approaches assign class labels more indirectly by learning $p(x, y)$, from which $p(y \mid x)$ (and hence the most likely class label) can be computed. To illustrate the difference, consider a logistic regression classifier (discriminative classifier) and Naive Bayes (generative classifier). For e-mail spam classification, a logistic regression classifier accepts a list of values (e.g., word frequencies from the message) and

Permission to make digital or hard copies of part or all of this work for personal or classroom use is granted without fee provided that copies are not made or distributed for profit or commercial advantage and that copies bear this notice and the full citation on the first page. Copyrights for third-party components of this work must be honored. For all other uses, contact the Owner/Author.

Copyright is held by the owner/author(s).

CHIIR '17, March 07-11, 2017, Oslo, Norway

ACM 978-1-4503-4677-1/17/03.

http://dx.doi.org/10.1145/3020165.3022172 outputs a label (e.g., whether the e-mail is spam). It can discover which terms are important in distinguishing spam but cannot determine the frequencies of terms in spam and non-spam documents. Yet Naive Bayes learns the probability of each word $x_{1}, x_{2}, \ldots, x_{n}$ in the vocabulary separately for each class label so could theoretically generate spam and non-spam documents by sampling from these distributions.

Query recommendation falls into a very similar framework as above. "Generative" approaches recommend queries by taking a user's context and modifying queries the user previously issued in the session, such as by substituting query terms or incrementally adding terms to a query [4]. As with Naive Bayes, they could generate any possible query with a compact representation, such as a language model over the possible terms. However, they make incremental refinements and do not take the necessary exploratory leaps often required of recommendations. As found by [11], recommendations are useful to people in the late stage of a long task, who issued several refinements and need a fresh start.

"Discriminative" query recommenders can offer more exploratory leaps more quickly. They select recommendations strictly from a query log - i.e., queries that have been issued in the past by users of a retrieval system. Some calculate explicit transition probabilities between queries in a log - as in a Markov model - to recommend likely subsequent queries from a user's context [3]. Others compute pairwise matrix similarities; [12] recommended queries that fell within a threshold similarity to balance relevance and diversity. These approaches rely on similarity measures or transition probabilities between queries to compute recommendations. Changing similarity thresholds can more heavily emphasize diverse or homogeneous recommendations. Moreover, the set of potential queries determined wholly by the input of users of an information retrieval system. However, such approaches can scale poorly, as they can require a matrix of up to size $|Q|$, where $Q$ is the number of queries and is exponential in the number of possible words.

The expansive query recommendation literature suggests a genuine need for query recommendations. Yet most work fails to consider the motivating task, even though there has been extensive work to theoretically model searchers' task structures [5] and to automatically extract searchers' tasks from web logs [6]. One recent query recommendation approach extracted the task - as a set of related queries - and topic - as a word distribution - and combined these to create tailored query recommendations [7]. Even then, such 
an approach does not immediately address the coverage of information needs of users. Hence, the following research questions are proposed to address at the doctoral consortium:

- To what extent, in terms of effectiveness and efficiency, can current discriminative and generative approaches to query recommendation be outperformed by a generative approach that generates queries directly from an information need?

- How can such an approach be evaluated in the absence of relevance judgments for documents?

\section{PROPOSED METHODOLOGY}

Query recommenders generally follow the following flow of information: 1) A recommender generates a background model of words, documents, queries, or some combination. 2) It uses the background model to map a user's log data queries and clicks - to a context model. 3) It determines (implicitly or explicitly) what information or documents a user should view next. 4) This determination is converted into a query recommendation. The same framework is followed here. As a proposed methodology, the author will attempt to show where discriminative methods such as query graphs fall short and where compact but smart generative methods are necessary. Attempts will be made to incorporate task and need information to create tailored recommendations and compare it to previous state-of-the-art recommenders.

\section{PREVIOUS WORK}

\subsection{Case for a Generative Approach}

Previous work from the author has suggested user queries can be less effective for recommendation than other approaches [9], calling for something beyond the discriminative recommender. In a Mechanical Turk task, participants guessed the query that generated a list of top 10 results, as on the first page of a Web search engine. The stimuli given in the Mechanical Turk task were real results from real queries issued to the Google Search API ${ }^{1}$, given as titles, URLs, and result snippets, with the underlying query hidden.

User guesses were then issued to the same API and compared against the original results and those of simple algorithms for guessing the query, given the same 10 results. A portion of the analysis is given in Figure 1. It shows that if the query length is known, an algorithm can greatly outperform users in guessing the underlying query. The best algorithm used a simple bag-of-words approach and output the most frequent words. Since an algorithm could guess the underlying query, it stands to reason that given a method for effectively determining a related set of relevant information (here as the top 10 results), an effective generative query recommendation can be given. Further recall that "discriminative" query recommenders largely select users' previous queries as candidates for recommendation. The results also suggest that even if a user is given an ideal set of information to search for, the user will issue a query that is less effective than that of a simple algorithm. This means users may issue suboptimal queries, which are in turn candidates for discriminative recommenders and can affect recommendation quality.

\footnotetext{
$\overline{{ }^{1} \text { https://www.google.com/cse/ }}$
}

\begin{tabular}{l|lll}
\multicolumn{1}{l}{} & Titles & Snippets & Titles+Snippets \\
\hline Users & 0.66 & - & - \\
Freq & 0.76 & 0.86 & 0.87 \\
Freq $(P)$ & 0.53 & 0.60 & 0.59 \\
Skipgram & 0.66 & 0.73 & 0.78 \\
Skipgram $(P)$ & 0.47 & 0.48 & 0.50
\end{tabular}

Figure 1: User and algorithmic performance in guessing queries, in Jaccard distance. (P) indicates a simple probabilistic model of query length, otherwise the query length is given. Results for the most frequent words (Freq) and most frequent skipgram.

\begin{tabular}{|l|c|c|c|c|}
\hline Algorithm & Tech. & Health & Ent. & Art \\
\hline Session & $\mathbf{1 1 . 6 3} \%$ & $14.74 \%$ & $7.89 \%$ & $7.35 \%$ \\
\hline ORTH & $9.97 \%$ & $\mathbf{2 6 . 3 4} \%$ & $\mathbf{1 6 . 4 2} \%$ & $\mathbf{4 0 . 6 8} \%$ \\
\hline LDA-SG & $9.21 \%$ & $20.03 \%$ & $8.39 \%$ & $31.02 \%$ \\
\hline
\end{tabular}

Figure 2: Recommendation performance of a discriminative approach (ORTH), our method [8] (LDA-SG), and user sessions (Session). Columns are for performance in Technology, Health, Entertainment, and Art search topics.

\subsection{Attempting a Generative Approach}

How, then, can a full generative pipeline be implemented: a background model, user context, a mapping to information needs, and ultimately a recommendation? The author approximated this notion of "information need" with a simpler topic-based approach in previous work [8]. For the background model, a Latent Dirichlet Allocation (LDA) topic model and a skip-gram model were pre-trained on a single web corpus, comprising 5 topics. LDA modeled a user's context - previous search queries and results - as a distribution over topics the user has seen so far. The "need" was approximated as a topic that was relevant but still diverged from the viewed topics. The skipgram model controlled phrases that were suggested as queries, so quality of queries would not degrade. This model completely generated queries by operating at the level of topic first and working down, using a simple (albeit naive) approach to modeling "need".

This approach was compared to an entirely discriminative approach from [12]. Users' sessions from a real lab study (searching on the same 5 Web corpus topics) were injected with recommendations. Some results are given in Figures 2 and 3. The proposed approach recommends queries that may give entirely unknown web pages that do not have a relevance score, which is also possible in a cold-start scenario with no relevance judgments. Therefore, traditional metrics such as Normalized Discounted Cumulative Gain (NDCG) will not work. Alternative approaches to precision and recall were hence used. A penalty score was used for precision: the percent of words or topics in recommended results that belonged to a different Web page topic than the one under recommendation. "Topicality" was measured at a URLbased level, using the Open Directory Project (ODP) mapping from URLs to topics. ${ }^{2}$ ODP was also used previously to mine query intention [2] and to evaluate recommenders. Recall was measured with the coverage of ODP topics. The new recommender can improve diversity, with some cost in relevance. In some cases, it outperforms a discriminative approach, showing promise with future improvements.

\footnotetext{
${ }^{2}$ http://www.dmoz.org
} 


\begin{tabular}{|l|c|c|c|c|}
\hline Unit of Measure & Tech. & Health & Ent. & Art \\
\hline Words & $0.06 \%$ & $1.11 \%$ & $1.15 \%$ & $0.83 \%$ \\
\hline ODP topics & $11.01 \%$ & $15.73 \%$ & $12.69 \%$ & $4.32 \%$ \\
\hline
\end{tabular}

Figure 3: Penalty scores for the combined LDA and skipgram approach.

\section{FUTURE PLAN}

\subsection{Future Methodology}

While work such as [7] shows that task and topic information can be combined in a hybrid model, [9] suggests query logs may not be effective as a direct source for query recommendations. A very coarse LDA model of "need" fueled recommendations and showed that topical recall can be improved with loss in precision with a simple model. Yet this shows that a more accurate method of determining relevant information or needs is required. For future research the author must address 1) improvements to the recommender, 2) datasets to use, and 3) methods for evaluation.

One direction for improvement is a better topic model that better separates topics, such as correlated LDA, which can create incremental improvements. For the data set, other established query logs with long sessions will be used in addition to the current data, such as the TREC Session Track $^{3}$. While this was used for the Mechanical Turk task to give a set of queries, it has not yet been used for the LDA-based query recommender. Lastly, evaluation should compare more baselines, including a generative one like [4] and a combined topical and query graph model like [7].

\subsection{Research Issues and Considerations}

There are several additional issues worth exploring to improve future research in this area. The first most pressing issue to address for this research is the evaluation metric. While ODP has been used in the past, traditional URLbased metrics such as NDCG are by far the most common. Yet for this type of generative recommender, the URLs in recommended queries may not have been manually annotated for relevance. Since lack of relevance judgments is also a problem for cold-start search tasks in general, it is a more universal problem. A possible direction of discussion is the development of a general, reasonable method of evaluation in addition to (or in place of) the previous metrics. How useful can such metrics be in the absence of relevance judgments, and how can they be empirically validated? On a related note, perhaps it would be better to use simulated users as in [1], evaluating which documents simulated users find useful instead of which relevant ones are discovered.

Another discussion point is potential components to add to a query recommender that 1 ) incorporate task and information need and 2) do not generate from a seed query, to test the viability of such a general framework. This requires refinements to models of task, need, and topic. One possibility is to model task and need as weighted combinations triples (as in the Resource Description Framework), to sample a subset of these for a recommendation, and to transform them into a query. Another complementary approach is to focus on modeling "needs" in a taxonomy, as [5] did with task facets, and optimize filling information gaps. Such an approach would also complement the precision-based per-

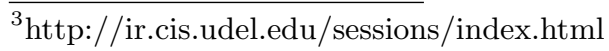

formance of [7]. These are suggestions are fueled by the belief that generative query recommenders that operate at the level of "information" rather than "queries" are a frontier worth exploring.

\section{ACKNOWLEDGMENTS}

This work has been supported by an ACM SIGIR Student Travel Grant. It was also funded by grants NSF IIS \#1423239 and IMLS \#LG-81-16-0025.

\section{REFERENCES}

[1] L. Azzopardi, D. Kelly, and K. Brennan. How query cost affects search behavior. In Proceedings of the 36th International ACM SIGIR Conference on Research and Development in Information Retrieval. ACM, 2013.

[2] R. Baeza-Yates, L. Calderón-Benavides, and C. González-Caro. The intention behind web queries. In Proceedings of the 13th International Conference on String Processing and Information Retrieval. Springer-Verlag, 2006.

[3] Q. He, D. Jiang, Z. Liao, S. C. H. Hoi, K. Chang, E.-P. Lim, and H. Li. Web query recommendation via sequential query prediction. In Proceedings of the 2009 IEEE International Conference on Data Engineering. IEEE Computer Society, 2009.

[4] R. Kraft and J. Zien. Mining anchor text for query refinement. In Proceedings of the 13th International Conference on World Wide Web. ACM, 2004.

[5] Y. Li and N. J. Belkin. An exploration of the relationships between work task and interactive information search behavior. Journal of the American Society for Information Science and Technology, 2010.

[6] C. Lucchese, S. Orlando, R. Perego, F. Silvestri, and G. Tolomei. Identifying task-based sessions in search engine query logs. In Proceedings of the Fourth ACM International Conference on Web Search and Data Mining. ACM, 2011.

[7] R. Mehrotra and E. Yilmaz. Terms, topics \& tasks: Enhanced user modelling for better personalization. In Proceedings of the 2015 International Conference on The Theory of Information Retrieval. ACM, 2015.

[8] M. Mitsui and C. Shah. Multi-word generative query recommendation using topic modeling. In Proceedings of the 10th ACM Conference on Recommender Systems. ACM, 2016.

[9] M. Mitsui and C. Shah. Query generation as result aggregation for knowledge representation. In Proceedings of the 50th Hawaii International Conference on System Sciences, 2017.

[10] A. Y. Ng and M. I. Jordan. On discriminative vs. generative classifiers: A comparison of logistic regression and naive bayes. In Advances in Neural Information Processing Systems 14. MIT Press, 2002.

[11] X. Niu and D. Kelly. The use of query suggestions during information search. Inf. Process. Manage., 2014.

[12] H. Vahabi, M. Ackerman, D. Loker, R. Baeza-Yates, and A. Lopez-Ortiz. Orthogonal query recommendation. In Proceedings of the rth ACM Conference on Recommender Systems. ACM, 2013. 